\title{
Effects of Dehydration on Brain Functioning: A Life-Span Perspective
}

\author{
Nathalie Pross \\ F. Hoffmann-La Roche Ltd., Basel, Switzerland
}

\author{
Keywords \\ Dehydration · Cognition · Mood · Adults · Children . \\ Adolescents · Elderly
}

\begin{abstract}
Background: In the last 10 years, there has been an increase in the publication of literature dealing with the effects of mild dehydration on cognition in healthy adults. Fewer studies, leading to less consistent data, involved other age groups. Summary: In healthy young adults refraining from drinking or participating in dehydration protocols, it was found that mild dehydration had no impact on performance, whereas the mood was widely impaired. Several studies have also been conducted in young children either as observational studies or as interventional studies. Nevertheless, methodological differences in (de)hydration monitoring, in cognitive assessments, and in the age/brain maturation of study participants, often resulted in contradictory findings regarding the cognitive functions impacted by (de)hydration. Although not consistent, these data showed that not only mood but also performance tend to be impaired by dehydration in children. Even if older adults are likely to be more vulnerable to dehydration than younger adults, very few studies have been conducted in this regard in this population. The results show that, like it is in children, cognition tends to be impaired when the elderly are dehydrated. Taken together, these studies suggest that dehydration has greater detrimental effects in vulnerable populations. Recent imaging
\end{abstract}

data suggest that the brain of children and elderly adults may have fewer resources to manage the effects of dehydration. Consequently, cognitive tasks may be more demanding for younger and older brains and performance more likely to be impaired in these populations, in comparison to young healthy subjects who have greater and more efficient resources.

(c) 2017 The Author(s)

Published by S. Karger AG, Basel

\section{From Severe Experiences of Dehydration to Everyday Life Experiences of Dehydration}

The link between dehydration and brain functioning has been well established in the field of medicine, particularly in elderly adults and young children. For different reasons, children and the elderly are vulnerable to dehydration and therefore represent a significant part of emergency hospitalizations (i.e., in the United States, dehydration is one of the 10 most frequent reasons leading to the hospitalization of the elderly [1]). In children, acute gastroenteritis and subsequent dehydration also represent an important percentage of pediatric emergency consultations [2]. Cognitive dysfunction is not listed among the diagnostic criteria in the guidelines for clinical assessment of dehydration in children [3]. Acute dehydration leading to hospitalization occurs often in very young children with acute diarrhea or with acute episodes of vomit-

\section{KARGER}

E-Mail karger@karger.com www.karger.com/anm

\section{The Author(s) \\ Published by S. Karger AG, Basel \\ Karger \\ Open access}

This article is licensed under the Creative Commons Attribution NonCommercial-NoDerivatives 4.0 International License (CC BYNC-ND) (http://www.karger.com/Services/OpenAccessLicense). Usage and distribution for commercial purposes as well as any distribution of modified material requires written permission.
Nathalie Pross

F. Hoffmann-La Roche Ltd.

Grenzacherstrasse 124

$\mathrm{CH}-4070$ Basel (Switzerland)

E-Mail nathalie.pross@ roche.com 
ing. These children are generally too young to speak fluently and therefore, the only cognitive sign of dehydration that is likely to be observed is a decrease in physical activity and/or an increased sleepiness [4]. Conversely, in the elderly, mental confusion, irritability, and sleepiness are frequently listed as the main alerting symptoms of dehydration and there is extensive literature dealing with dehydration and delirium in this population [5]. In these extreme situations of dehydration, the brain is obviously impacted even if there are no studies that have described and quantified this effect on cognitive functioning.

The effects of severe dehydration have been better characterized in sport and military scenarios that studied the effects of fluid deprivation in healthy adult populations. Most of these studies were conducted in extreme conditions combining heat exposure and exercise to accentuate the effects of dehydration, with the result that dehydration clearly impaired cognitive performance (see [6] for a review). However, inducing dehydration using heat and/or physical activity contains a methodological confounder, since each stressor affects cognition independently of dehydration [7]. In addition, these data are often inconsistent regarding which cognitive functions are impaired and at which level of dehydration the first impairing effects occur (for a review see [8]). Thus, these studies demonstrated the impairing effects of severe dehydration on cognitive functioning, but this effect has not been clearly characterized. These data are also difficult to extend to daily life situations in which severe dehydration occurs rarely and in which athletes and soldiers do not represent the main population.

Therefore, in the past 15 years, the interest in the topic of dehydration and cognition has switched from medicine studying specific populations (patients, athletes, soldiers) to physiology and psychology examining the effects of dehydration on brain functions in healthy volunteers. The first studies focused on the effects of moderate to severe dehydration on a wide range of cognitive functions, including subjective feelings [9-11], but more recently interest has switched over to the effects of mild dehydration on healthy people while performing everyday activities.

\section{Mild Dehydration and Cognition in Adults}

One of the first studies dealing with mild dehydration in healthy volunteers during daily activity used moderate exercise in a moderate-warm environment to achieve mild dehydration $[12,13]$. Twenty-six men (mean age: $20.0 \pm$ 0.3 years) and 25 women (mean age: $23.0 \pm 0.6$ years) participated in this 3-period, crossover, randomized, placebo-controlled study. Of particular interest was the positive control condition in which dehydration was achieved by the combination of a diuretic and exercise. To further blind the experimental conditions, a pill (either diuretic or placebo) was also administered prior to each test session. One session consisted of an exercise-induced dehydration plus placebo condition, the second session involved an exercise-induced dehydration plus diuretic condition, and the control condition was described as a euhydration plus placebo condition. Cognition was assessed 20 min after 3 exercise sessions in a quiet room $\left(23^{\circ} \mathrm{C}\right)$. These testing sessions lasted about $45 \mathrm{~min}$ and included computerized tasks, which assessed memory, vigilance, speed of processing, and several mood and physiological states. The results showed that mild levels of dehydration differently affected men and women. While few changes in cognitive performance have been observed in both genders, women exhibited a variety of adverse changes in key mood states (i.e., fatigue), and more headaches and concentration difficulties, while the men did not. The authors concluded that women are more sensitive to the effects of mild dehydration than men. This dissociation between the effect of mild dehydration on performance and mood in young healthy women has been confirmed by another group of researchers. In a study evaluating the effect of an acute fluid restriction on mood and cognition, 20 young healthy women (mean age: $25 \pm 3.5$ years) underwent a randomized 2 -period crossover study [14]. During the fluid restriction period, participants were asked to refrain from drinking for $24 \mathrm{~h}$. During the control condition, water intake (a total of $2 \mathrm{~L}$ ) was allowed at fixed time points and was dissociated from meals so that eating was similar for both periods. Mood and cognitive assessments were scheduled at different time points through the day in order to mimic a typical working day. Results showed that fluid deprivation had deleterious effects on several mood aspects and that these effects were observable only after a few hours of fluid deprivation. Overall, the earliest and the most important effects were observed on sleep/wake parameters. The participants reported increased sleepiness and fatigue during the fluid deprivation condition. They also were less vigorous and less alert, more confused, less calm, and less happy during the fluid deprivation condition, when compared to the control condition. However, dehydration did not affect all cognitive functions. Cognitive performance as assessed by attentional, memory, vig- 
ilance, and speed of processing tasks was not impaired, even after more than $20 \mathrm{~h}$ of fluid deprivation. The effects of rehydration were also measured; mood was reassessed at the end of each study period, after ad libitum fluid intake. The results indicated that most of the mood impairments induced by fluid deprivation were reversed by this acute water intake. However, no improvement in fatigue, vigor, and calmness was reported after this water intake, indicating that a $24 \mathrm{~h}$-fluid deprivation may have longerterm effects on the emotional state.

Even if obtained in well-controlled conditions, these results are difficult to extend to daily living situations because a $24 \mathrm{~h}$-fluid deprivation is not likely to occur frequently in the main population. This conclusion led to a more realistic experimental design in which subtle changes of hydration status were induced. In this study, habitual high-volume ( $\geq 2 \mathrm{~L} /$ day) and low-volume $(<1.2 \mathrm{~L} /$ day $)$ drinkers were asked to respectively decrease (i.e., to $1 \mathrm{~L}$ per day) and increase (i.e., to $2.5 \mathrm{~L}$ per day) their daily water intake during 3 controlled intervention days [15]. The effects of these changes in habitual water intake were measured on mood and sensations at several time points during the day. Self-reported assessments included mood scales (POMS, [16]; emotional visual analogue scale, [17]) and physiological sensation scales (thirst visual analogue scale, Karolinska sleepiness scale, [18]). The results showed that increased daily water intake had beneficial effects on several mood and sensation assessments in habitual low-volume drinkers. Low-volume drinkers indicated less fatigue, less confusion, and less thirst; they tended also to be less asleep after the switch toward increased habitual water intake. On the other hand, decreased daily water intake had detrimental effects on mood ratings in habitual high-volume drinkers. After the switch toward restricted water intake, high-volume drinkers indicated being thirstier, less calm, less content, less vigorous, and reported lower positive emotions. These results demonstrated that not only moderate-tosevere dehydration has a significant impact on daily cognition, but even mild dehydration leads to significant effects on everyday functioning. In addition, this study also demonstrated that increasing habitual fluid intake has a beneficial effect on the emotional state and the overall well-being of the individual.

Even if consistent, the results obtained in young adults cannot be generalized to other populations such as the elderly and children. Indeed, from a cognitive perspective, young children and older adults differ in terms of cognitive capacity, strategy effectiveness, and overall efficiency (i.e., compensatory mechanisms to maintain their performance level have been evidenced in the elderly, [19]; strategic behaviors are constantly developing in young children and adolescents $[20,21])$.

\section{Dehydration and Cognition in Children}

The interest to study the effects of dehydration on cognitive performance in children is a recent trend. The first studies were published less than 15 years ago. Since then, the number of dehydration studies has grown because children are an at-risk population. Indeed, young children rely often on adults to have access to fluids. They are also more vulnerable to dehydration due to differences in body-cooling mechanisms and to differences in thirst sensations when compared to adults [22].

One of the first studies dealing with hydration and cognition in schoolchildren was performed in the Israeli desert where ambient temperature was $35^{\circ} \mathrm{C}$ and class room temperature was $30^{\circ} \mathrm{C}$. Fifty-eight children aged 10-12 years participated in this noninterventional study [23]. Urine samples were collected in the morning and at noon. At the same time points, the children were also asked to complete 5 cognitive tests that assessed their working memory, short-term memory, executive functions, and visual-processing abilities. The urinary osmolality measures revealed that 32 children were dehydrated in the morning (i.e., morning urine osmolality was above $800 \mathrm{mOsm} / \mathrm{kg} \mathrm{H}_{2} \mathrm{O}$ ). However, no significant difference was evidenced in the cognitive performance assessed in the morning among the children when hydration status was considered. The noon urine samples revealed that among the 32 children that were dehydrated in the morning, 26 were still dehydrated at noontime. A significant impact of dehydration on performance was observed while conducting the mid-day cognitive tests. Well-hydrated children tended to perform better than dehydrated children, especially in working memory measures. The authors concluded that dehydration was a common phenomenon in schoolchildren and that this may impair their cognitive functioning. One may observe that these study results cannot be generalized to all schoolchildren because this study was conducted in an arid climate, resulting in a large percentage of dehydrated children.

A more recent study performed in France [24] with a greater sample $(N>500)$ reported similar data regarding the number of children that are dehydrated in the morning when arriving at school. Indeed, $62.2 \%$ had a urine osmolality value above $800 \mathrm{mOsm} / \mathrm{kg} \mathrm{H}_{2} \mathrm{O}$. Unfortunately, cognitive performance was not assessed, but a series of
Pross 
interventional studies confirmed the preliminary finding of Bar-David et al. [23]. One of these studies aimed to examine whether providing schoolchildren with water during class hours has an effect on their school performance [25]. Memory and attentional abilities were assessed in 40 children (mean age: 8.7 years). These cognitive assessments were done on 2 consecutive days, in controlled, counterbalanced experiments (i.e., constant room temperature and approximately same time of testing). The only difference between experimental sessions was that one day the participants were asked to drink $300 \mathrm{~mL}$ of water, 25 min prior to the cognitive assessments; on another day, no water was provided prior to the cognitive testing. The results showed that attentional abilities were not impacted by water consumption but that children performed better on the memory task the day they were provided with water prior to the testing. Another interventional study confirmed that providing water to schoolchildren during class hours has a beneficial effect on cognitive functioning [26]. In that study, cognitive performance (i.e., visual memory, attention and visuomotor abilities) was assessed in 2 groups of children (mean age: 8.6 years). One group was provided with $250 \mathrm{~mL}$ water, with the instruction to drink as much as desired. The other group of children was not provided with water and was considered to be the control group. Subjective thirst sensation was assessed in all children. The results indicated that the mean consumption of water was $212 \mathrm{~mL}$ in the children who were provided with water. In this group, the subjective feeling of thirst was significantly lower in comparison to that of the control group. When cognitive performance was considered, a beneficial effect of water drinking was observed on visual attention and memory tasks but not on psychomotor performance. This study was replicated by the same group of researchers [27], employing the same cognitive tasks as in the previous study but providing 500 $\mathrm{mL}$ of water. The results showed that by following a mean consumption of $409 \mathrm{~mL}$ of water, the children had better attentional and visual search performance than the control group. However, no hydration effect was observed on the memory function and psychomotor tasks.

More recently, a correlation between habitual water intake and high-level attentional control processes has been observed [28] in an observational study (i.e., no intervention on fluid intake). Total water intake was defined as the average daily total water plus the water in food, recorded through 3-day food and beverage records. Sixty-three children (mean age: $8.6 \pm 0.3$ years) were asked to perform a high-level attentional control task: the adapted-for-children modified Flanker task [29]. The

Effects of Dehydration on Brain

Functioning participants were divided into low-volume and high-volume drinkers according to their median total water intake $(1,046 \mathrm{~mL} /$ day $)$ and their cognitive performance was analyzed according to their drinking status. The results showed that habitual water intake was significantly correlated with high-level cognitive control abilities. The low drinkers had worse reaction times and committed more inhibition errors during the most demanding condition. Thus, the beneficial hydration effect was not only observed for speed of processing but also for performance accuracy, both being implicated in numerous daily activities and especially in the school activities of children.

\section{Dehydration and Cognition in the Elderly}

Older adults are also considered to be an at-risk population regarding dehydration. Decreased perception of thirst leading to involuntary dehydration has been welldescribed in older adults [30]. In addition, total body water decreases in the elderly, mainly due to changes in adipose tissue and muscle mass, and to a less effective renal response to mild water deprivation. Like children, older adults often rely on others for access to water. Although the risk of dehydration in cognitively impaired or older dependent adults is widely recognized, less attention has been paid to the effects of mild dehydration during everyday life, as attested by the few studies published in this domain. To the best of our knowledge, only 2 studies have examined the effects of mild dehydration on cognitive performance in older community-dwelling adults. One [31] involved 28 healthy independent-living adults (22 females; mean age $63.7 \pm 3.5$ years). The participants were asked to complete the following standardized cognitive tests: the trail making test [32], the Repeatable Battery for the Assessment of Neuropsychological Status [33], and the grooved pegboard test [34]. Hierarchical regression analyses examined whether hydration status accounted for significant variance in 2 main cognitive processes: psychomotor processing speed and memory performance. Even after controlling for the effects of blood pressure, age, and education on cognition, poorer hydration was statistically associated with poorer memory and psychomotor speed slowing. The authors [35] tried to confirm these results in a sample of 21 postmenopausal women (mean age $60.3 \pm 8.0$ years), selected from a larger study including both genders, examining the relationship between stress and cognition. The cognitive function tests were slightly different from those used in their previous study, as they focused on declarative memory by us- 
ing the auditory verbal learning test (AVLT) and on working memory by using the auditory consonant trigrams (ACT, [36]). The results showed findings that were consistent with the previous study. In these healthy older community-dwelling women, a significant relationship existed between mild dehydration and cognitive functions. Better hydration was significantly correlated with a greater number of correctly recalled words in the AVLT and better working memory span in the ACT. However, when diastolic blood pressure was entered into the statistical models, these relationships were attenuated. This suggested that lower blood pressure is related to more efficient declarative memory and working memory abilities. The authors concluded that both hydration status and blood pressure should be taken into account when interpreting the cognitive performance of elderly adults.

\section{Dehydration and Cognition in Adolescents}

To the best of our knowledge, only one study has examined the effects of dehydration on cognitive functions in non-athletes and healthy adolescents [37]. This may be explained by the fact that studying cognition in this population is complicated, due to large inter-individual variability in brain maturation (and consequently in cognitive functioning) within this age group [38]. Nevertheless, the only published study is of particular interest because the authors investigated the effect of dehydration on the brain via functional MRI (fMRI). In this cross-over, randomized study, 10 healthy adolescents ( 5 boys, mean age: 16.8 years) were asked to perform two 90 -min periods of physical exercise. In the control condition, the exercise protocol (50 min cycling, $10 \mathrm{~min}$ rest [seated recovery], 20 min cycling, 10 min rest) was performed in light sport clothing. During the dehydration condition, the participants were asked to wear 3 layers of clothing to accentuate sweating. This thermal exercise dehydration protocol aimed to decrease body mass by $1-2 \%$. In both study conditions, body temperature and water intake were well controlled. Heart rate was monitored and urine osmolality was measured. Subjective sensations and weight were monitored before and after exercise. Structural, functional, and arterial spin labelling (ASL) imaging measures were performed pre and post exercise in both study conditions (control and dehydration). The fMRI scan was associated with an executive functioning measure, the Tower of London. This task requires high-level cognitive functions (mainly planning) and is recognized to involve frontal lobe resources. The results showed that the par- ticipants were normally hydrated before the exercise protocol in both conditions. The thermal exercise dehydration condition led to greater dehydration levels, as attested by body mass, heart rate, and body temperature measures. Subjective sensation ratings were equivalent between both study conditions and cognitive performance, as measured by the Tower of London task was not impaired by dehydration. Interestingly, fMRI data showed an increase in the frontoparietal BOLD (bold-oxygenlevel-dependent) signal while dehydrated in comparison to the control condition. Thus, in the dehydrated condition, the adolescent brain exerted greater effort to achieve the same performance as in the control condition. This is known as a brain-compensatory mechanism and has previously been described in healthy elderly adults [39]. ASL data indicated no changes in cerebral resting state perfusion during dehydration. However, a significant correlation was observed between the percentage change of body mass and the percentage change of brain ventricular volume while dehydrated. This increase in the volume of the lateral ventricles indicates that dehydration is associated with significant effects on brain volume.

\section{Conclusions}

Taken together, these data indicate that mild dehydration differentially affects brain functioning in healthy subjects according to age. Cognitive functions such as attention, memory, or executive functions are impaired in mildly dehydrated schoolchildren. Even if the data are not completely consistent regarding the impacted cognitive functions, the performance of schoolchildren is systematically impaired when hydration levels are manipulated. Cognitive performance is impaired in dehydrated schoolchildren but is improved when water is provided during the class. In the only study performed on healthy, non-athletic adolescents, no detrimental effect on cognitive performance was observed after mild dehydration. Nevertheless, imaging data showed that even if dehydrated adolescents were able to maintain their habitual performance level, dehydration effects were observed in the structure and the functioning of their brain.

In adults, data showed a clear and consistent mood worsening during mild dehydration. This mood worsening is mainly characterized by sleepiness complaints and a significant impairment of well-being. When cognitive performance is considered, data are less consistent. Some studies showed impairments of cognitive functions, whereas cognitive performance was preserved in other
Pross 
studies. However, when examined in detail, it appears that performance is often impaired when dehydration is achieved by combining fluid restriction with heat or exercise. When dehydration is induced by fluid deprivation only, healthy adults appear to be able to maintain performance. In elderly adults, the few studies that have been performed indicated that mild dehydration impairs cognitive performance, as in young children. These results have to be confirmed and research also should be extended to the relationship between dehydration and mood; in elderly individuals, no data are available to date.

This review presents some evidence regarding a deleterious effect of dehydration on the cognitive performance of children and older adults, whereas no effects were visible in non-elderly adults and adolescents. Regarding mood, significant and consistent changes have been observed in adults when hydration levels were manipulated. In other age groups, there are not enough data to support conclusions. The study using functional magnetic imaging technology in adolescents brings an explanation to this differential effect of dehydration on brain functioning across life span. Imaging data revealed that adolescents are able to maintain their habitual level of cognitive performance when dehydrated, but this performance maintenance has a cognitive cost, evidenced by the greater neuronal activity in frontoparietal regions. In other terms, to achieve a same level of performance, the brain exerted greater effort when dehydrated. This may be possible in individuals, such as adolescents and healthy adults, who have enough resources or cognitive reserve (for a review of this concept, see [40, 41]). More vulnerable persons (e.g., children and elderly adults) may have fewer resources or cognitive reserve to manage the effects of dehydration. Consequently, cognitive tasks may be more demanding for younger and older dehydrated brains and performance is more likely to be impaired in these populations. One may also suggest that this influences mood worsening in healthy adults. Indeed, when mildly dehydrated, healthy adults are able to maintain their habitual level of performance but they exhibit higher levels of fatigue, worsening of well-being, and are less clear-minded. These effects on mood may reflect the greater neuronal activity that is exerted to compensate the effect of mild dehydration. In future research, the use of imaging techniques may help researchers to better understand and characterize the effects of dehydration on brain functioning and to further investigate the hypothesis of a neuronal compensation for dehydration by the less vulnerable brains.

This review also showed that there is a dramatic need for more studies involving the elderly and adolescent participants. Lack of data in older adults may be explained by the greater difficulty of controlling baseline inter-individual differences such as comorbidity and comedication. The reasons for the lack of adolescent data likely involve inter-individual differences of cognition due to differences in brain maturation during a period of life characterized by emotional lability. Lastly, a real need exists for more methodological standardization, especially in studies that observe children and adolescents. This need includes improvement of study designs (i.e., necessity to have baseline assessments to have a "placebo" intervention when using parallel groups in intervention studies) and use of validated cognitive tests.

\section{Disclosure Statement}

The author received travel expenses and registration fee from Danone Research to attend the $\mathrm{H} 4 \mathrm{H}$ Scientific Conference.

\section{References}

1 Sfera A, Cummings M, Osorio C: Dehydration and cognition in geriatrics: a hydromolecular Hypothesis. Front Mol Biosci 2016;3:18.

2 Whitney RE, Santucci K, Hsiao A, Chen L: Cost-effectiveness of point-of-care testing for dehydration in the pediatric ED. Am J Emerg Med 2016;34:1573-1575.

3 Steiner MJ, DeWalt DA, Byerley JS: Is this child dehydrated? JAMA 2004;291:2746-2754.

4 Lee GO, Richard SA, Kang G, Houpt ER, Seidman JC, Pendergast LL, Bhutta ZA, Ahmed T, Mduma ER, Lima AA, Bessong P, Jennifer MS, Hossain MI, Chandyo RK, Nyathi E, Lima IF, Pascal J, Soofi S, Ladaporn B, Guerrant RL, Caulfield LE, Black RE, Kosek MN; MAL-ED
Network Investigators: A comparison of diarrheal severity scores in the MAL-ED multisite community-based cohort study. J Pediatr Gastroenterol Nutr 2016;63:466-473.

5 Rosen T, Connors S, Clark S, Halpern A, Stern ME, DeWald J, Lachs MS, Flomenbaum N: Assessment and management of delirium in older adults in the emergency department: literature review to inform development of a novel clinical protocol. Adv Emerg Nurs J 2015;37:183-196.

6 Lieberman HR: Hydration and cognition: a critical review and recommendations for future research. J Am Coll Nutr 2007;26:S555S561.
7 Lieberman HR: Hydration and human cognition. Nutr Today 2010;45:S33-S36.

8 Popkin BM, D’Anci KE, Rosenberg IH: Water, hydration, and health. Nutr Rev 2010;68:439458

9 Shirreffs SM, Merson SJ, Fraser SM, Archer DT: The effects of fluid restriction on hydration status and subjective feelings in man. $\mathrm{Br}$ J Nutr 2004;91:951-958.

10 Szinnai G, Schachinger H, Arnaud MJ, Linder L, Keller U: Effect of water deprivation on cognitive-motor performance in healthy men and women. Am J Physiol Regul Integr Comp Physiol 2005;289:R275R280.
Effects of Dehydration on Brain

Functioning
Ann Nutr Metab 2017;70(suppl 1):30-36 DOI: $10.1159 / 000463060$ 
11 Petri NM, Dropulic N, Kardum G: Effects of voluntary fluid intake deprivation on mental and psychomotor performance. Croat Med J 2006;47:855-861.

12 Armstrong LE, Ganio MS, Casa DJ, Lee EC, McDermott BP, Klau JF, Jimenez L, Le Bellego L, Chevillotte E, Lieberman HR: Mild dehydration affects mood in healthy young women. J Nutr 2012;142:382-388.

13 Ganio MS, Armstrong LE, Casa DJ, McDermott BP, Lee EC, Yamamoto LM, Marzano S, Lopez RM, Jimenez L, Le Bellego L, Chevillotte E, Lieberman HR: Mild dehydration impairs cognitive performance and mood of men. Br J Nutr 2011;106:1535-1543.

14 Pross N, Demazières A, Girard N, Barnouin R, Santoro F, Chevillotte E, Klein A, Le Bellego L: Influence of progressive fluid restriction on mood and physiological markers of dehydration in women. Br J Nutr 2013;109: 313-321.

15 Pross N, Demazières A, Girard N, Barnouin R, Metzger D, Klein A, Perrier E, Guelinckx I: Effects of changes in water intake on mood of high and low drinkers. PLoS One 2014;9: e94754.

16 McNair DM, Lorr M, Droppleman LF: Manual of the Profile of Mood State. San-Diego, Educational and Industrial Testing Service, 1981.

17 Lees N, Lloyd-Williams M: Assessing depression in palliative care patients using the visual analogue scale: a pilot study. Eur J Cancer Care (Engl) 1999;8:220-223.

18 Akerstedt T, Gillberg M: Subjective and objective sleepiness in the active individual. Int J Neurosci 1990;52:29-37.

19 Erk S, Spottke A, Meisen A, Wagner M, Walter H, Jessen F: Evidence of neuronal compensation during episodic memory in subjective memory impairment. Arch Gen Psychiatry 2011;68:845-852.

20 Piaget J: La Naissance de l'intelligence chez l’Enfant, Delachaux et Niestlé, 1936.
21 Lehto JE, Juujärvi P, Juujärvi LX, Pulkkinen L: Dimensions of executive functioning: evidence from children. Br J Dev Psychol 2003; 21:59-80.

22 D'Anci KE, Constant F, Rosenberg IH: Hydration and cognitive function in children. Nutr Rev 2006;64(10 pt 1):457-464.

23 Bar-David Y, Urkin J, Kozminsky E: The effect of voluntary dehydration on cognitive functions of elementary school children. Acta Paediatr 2005;94:1667-1673.

24 Bonnet F, Lepicard EM, Cathrin L, Letellier C, Constant F, Hawili N, Friedlander G: French children start their school day with a hydration deficit. Ann Nutr Metab 2012;60:257263.

25 Benton D, Burgess N: The effect of the consumption of water on the memory and attention of children. Appetite 2009;53:143-146.

26 Edmonds CJ, Burford D: Should children drink more water?: the effects of drinking water on cognition in children. Appetite 2009; 52:776-579.

27 Edmonds CJ, Jeffes B: Does having a drink help you think? 6-7-Year-old children show improvements in cognitive performance from baseline to test after having a drink of water. Appetite 2009;53:469-472.

28 Khan NA, Raine LB, Drollette ES, Scudder MR, Cohen NJ, Kramer AF, Hillman CH: The relationship between total water intake and cognitive control among prepubertal children. Ann Nutr Metab 2015;66(suppl 3):S38S41.

29 Scudder MR, Khan NA, Lambourne K, Drollette ES, Herrmann SD, Betts JL, Washburn RA, Donnelly JE, Hillman CH: Cognitive control in preadolescent children with risk factors for metabolic syndrome. Health Psychol 2015;34:243-252

30 Kenney WL, Chiu P: Influence of age on thirst and fluid intake. Med Sci Sports Exerc 2001; 33:1524-1532.
31 Suhr JA, Hall J, Patterson SM, Niinistö RT: The relation of hydration status to cognitive performance in healthy older adults. Int J Psychophysiol 2004;53:121-125.

32 Reitan RM: Validity of the trail making test as an indicator of organic brain damage. Percept Mot Skills 1958;8:271-276.

33 Randolph C: Repeatable Battery for the Assessment of Neuropsychological Status. San Antonio, Psychological Corporation, 1998.

34 Matthews CG, Klove H: Instruction Manual for the Adult Neuropsychology Test Battery. Madison, University of Wisconsin Madison, 1964.

35 Suhr JA, Patterson SM, Austin AW, Heffner KL: The relation of hydration status to declarative memory and working memory in older adults. J Nutr Health Aging 2010;14:840-843.

36 Strauss E, Sherman EMS, Spreen O: A Compendium of Neuropsychological Tests: Administration, Norms, and Commentary, ed 3. New York, Oxford University Press, 2006.

37 Kempton MJ, Ettinger U, Foster R, Williams SC, Calvert GA, Hampshire A, Zelaya FO, O'Gorman RL, McMorris T, Owen AM, Smith MS: Dehydration affects brain structure and function in healthy adolescents. Hum Brain Mapp 2011;32:71-79.

38 Giedd JN, Rapoport JL: Structural MRI of pediatric brain development: what have we learned and where are we going? Neuron 2010;67:728-734.

39 Mathis A, Schunck T, Erb G, Namer IJ, Luthringer $\mathrm{R}$ : The effect of aging on the inhibitory function in middle-aged subjects: a functional MRI study coupled with a color-matched Stroop task. Int J Geriatr Psychiatry 2009;24: 1062-1071.

40 Barulli D, Stern Y: Efficiency, capacity, compensation, maintenance, plasticity: emerging concepts in cognitive reserve. Trends Cogn Sci 2013;17:502-509.

41 Stern Y: An approach to studying the neural correlates of reserve. Brain Imaging Behav 2016, Epub ahead of print. 